\title{
Structural Analyses of AC4 Protein of Sri Lankan Cassava Mosaic Virus
}

\author{
Sohini Gupta ${ }^{1}$, Sayak Ganguli ${ }^{2 *}$, Protip Basu ${ }^{2}$, Abhijit Datta ${ }^{3}$ \\ ${ }^{1}$ PG Department of Botany, Barasat Government College, Barasat \\ ${ }^{2}$ DBT-Centre for Bioinformatics, Presidency University, Kolkata \\ ${ }^{3}$ Department of Botany, Jhargram Raj College, Jhargram \\ *E-mail address: sohinigupta18@gmail.com
}

\begin{abstract}
RNA silencing is one of the important phenomenon in plant defense mechanism, it actively protect host plants against viral infections. Existing viruses must have developed counter defense strategies to survive this arms race. Such counter defense strategy is the viral silencing suppressor (VSRs) which have been reported to directly interfere with the various steps leading to the interference of viral RNAs. Most identified VSRs are multifunctional, besides being RNA-silencing suppressors, they often perform essential roles by functioning as coat proteins, helper components for viral transmission, replicases and movement proteins, proteases or transcriptional regulators. One such identified VSR is AC4 of Sri Lankan cassava mosaic virus strain. Trivial knowledge about the structure -function relationship of this VSR leads to this work, where we focus on the structure generation by modelling to identify the mode of interactions with the various effector molecules of the silencing pathways. Structural analyses have been performed to screen interacting residues. Results indicate conserved structural features which signify propensity of functional interactions and further shows that this VSR can be a potent tool for the analysis of RNA silencing mechanisms and the relationships between different silencing pathways and VSRs.
\end{abstract}

Keywords: Viral silencing suppressor proteins; Geminivirus; post transcriptional gene silencing

\section{INTRODUCTION}

Geminiviruses are one of the most important and the largest group of viruses that possesses much smaller genome than most of the other viruses. Fauquet et.al. (2003) has proposed a four fold classification of the group based on the genome, nature of insect vectors and host ranges. Geminiviruses are heavily dependent on the host replication and transcription machineries for their gene expression. (Gutierrez 1999; Hanley-Bowdoin et al., 1999). Pilartz and Jeske (1992) have observed that DNA-A and DNA-B are the two genomic components of African cassava mosaic virus of which six genes are encoded by DNA-A, two in the virus sense: $A V 1$, which codes for the coat protein (CP), and $A V 2$, whose specific role is not yet known, in the complementary sense, rest of the four genes reside, which comprise of $A C 1$ that codes for replication-associated protein (Rep); $A C 2$ and $A C 3$, which code for the transcription protein and the replication enhancer protein, and AC4. AC4 has been reported to have divergent biological functions, for example in the infectivity of several bipartite geminiviruses 
(Elmer et al. 1988; Etessami et al. 1991; Hoogstraten et al. 1996; Pooma and Petty 1996; Sung and Coutts 1995). According to Vanitharani and associates (2004) and Gupta et.al. (2014), African cassava mosaic virus (ACMV) AC4 suppresses posttranscriptional gene silencing (PTGS). Evidences show that AC4 may selectively bind the single stranded siRNAs including miRNAs. (Chellappan, P., Vanitharani, R. and Fauquet, C.M. 2005). The following strains of cassava-infecting bipartite begomoviruses, namely African cassava mosaic virus from Cameroon (ACMV-[CM]), East African cassava mosaic Cameroon virus (EACMCV), EACMV from Uganda (EACMV-[UG]), Sri-Lankan cassava mosaic virus (SLCMV) and Indian cassava mosaic virus (ICMV) were recently shown to elicit the PTGS system in infected plants with the production of virus-specific siRNAs (Chellappan, P., Vanitharani, R. \& Fauquet, C. M. (2004), Vanitharani, R., Chellappan, P. \& Fauquet, C. M. 2005). The identification of AC4 indicates that this protein play different roles and consequently might target various steps in the silencing pathway, or may possibly interact with different host proteins and furthermore contribute to the suppression of RNA silencing. This work focuses on the structural analyses of the Srilankan Cassava Mosaic virus AC4 protein.

\section{MATERIALS AND METHOD}

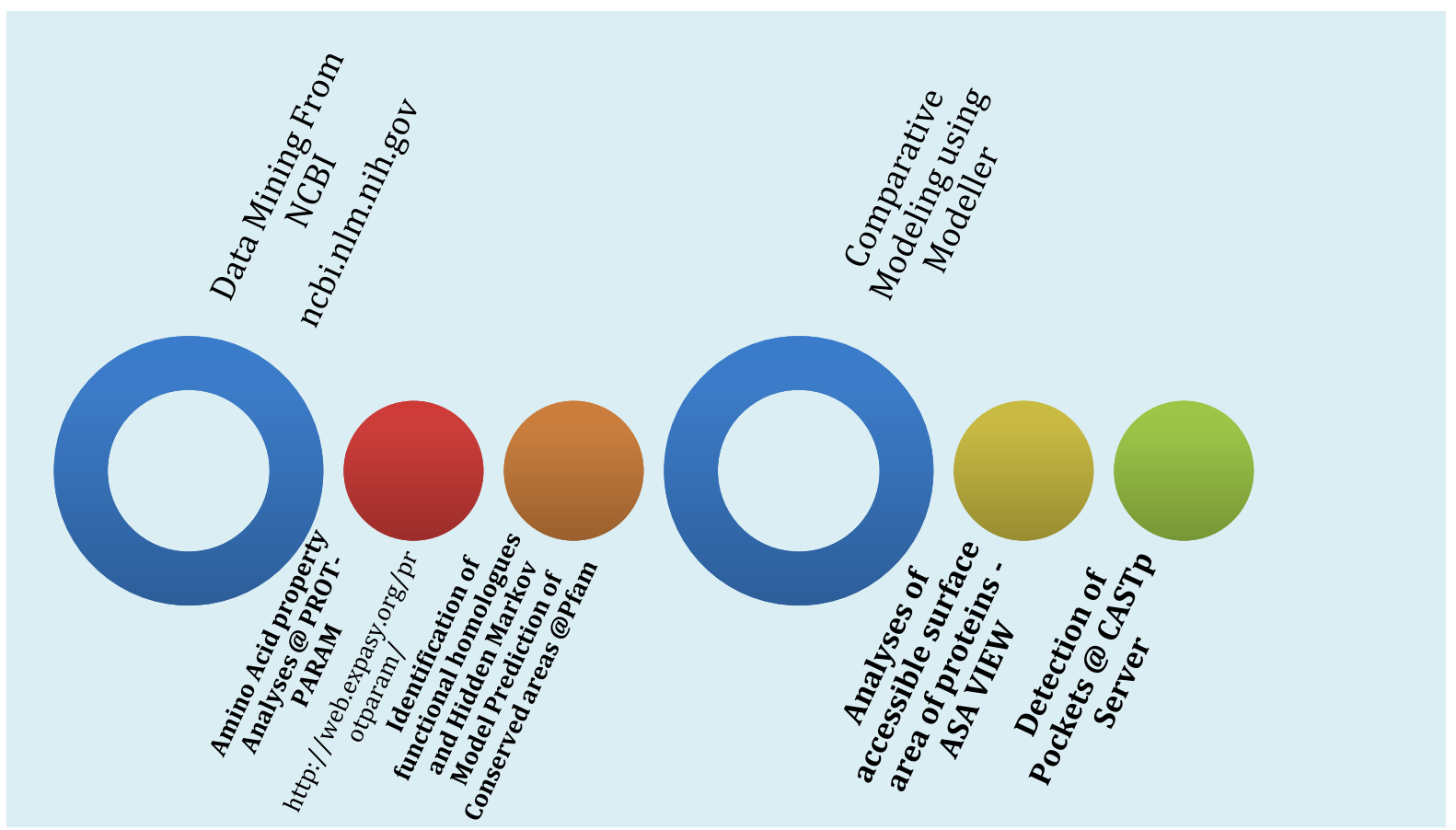

Fig. 1. Flowchart of work.

The above workflow has been previously used for the analyses of various protein structures across all domains of life (Ganguli et. al. 2013; Gupta et.al. 2013). The following colour scheme was used for the identification of different accessible surface areas of the amino acids of the protein -

Blue -> Positive charged residues $(R, K, H)$ 
Red $->$ Negative charged residues $(D, E)$

Green-> Polar uncharged residues ( $G, N, Y, Q, S, T, W)$,

Yellow -> Cysteine

Gray -> Hydrophobic residues (All others)

\section{RESULTS AND DISCUSSION}

Structural analyses of the AC4 viral silencing suppressor reveals predominant helical segments joined together by coiled coil structures. These secondary structures are well represented in the 3D model and are accompanied by a single antiparallel beta sheet region (Fig 1). Five different conserved positions (Fig 2) were detected from the multiple sequence alignment that was performed with three positions corresponding to serine residues which are placed in the outermost ring of the spiral plot(Fig 1) obtained indicating a role towards serving as possible sites for phosphorylation. Furthermore serine was also found to be the most frequently represented residue in the sequences that were studied.

Pocket identification revealed the presence of a single open mouthed pocket comprised of over 50 amino acid residues. This pocket contained all the five identified conserved residues (fig 1).
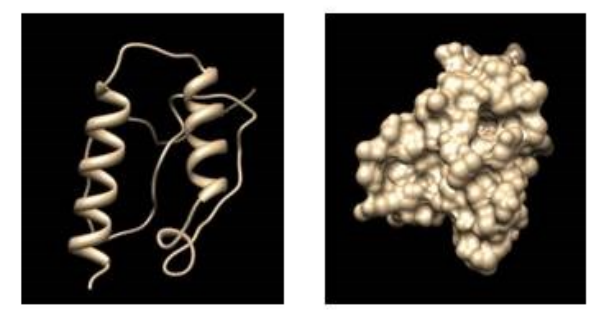

NAME OF THE AMINO ACID RESIDUE
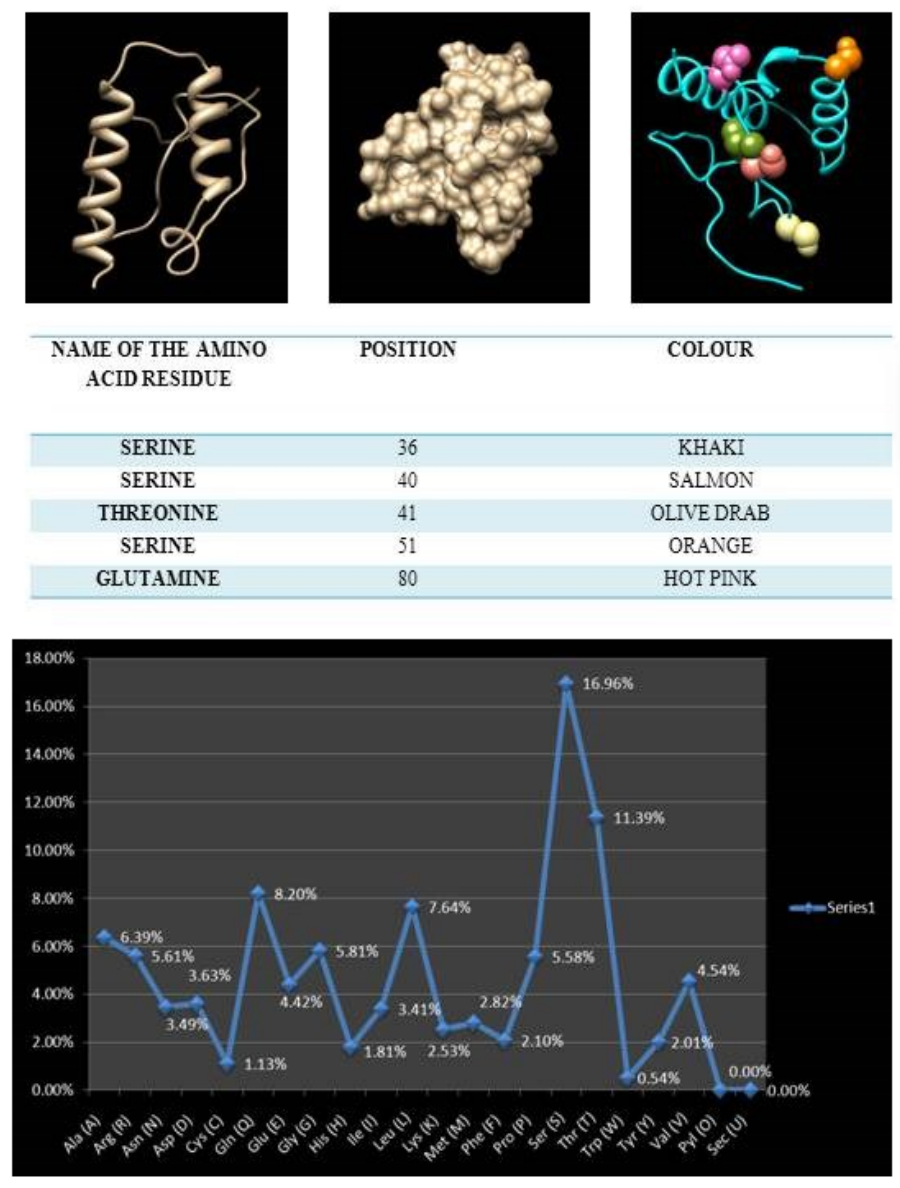

COLOUR

KHAKI LIVE DRAB ORANGE THREONINE SERINE
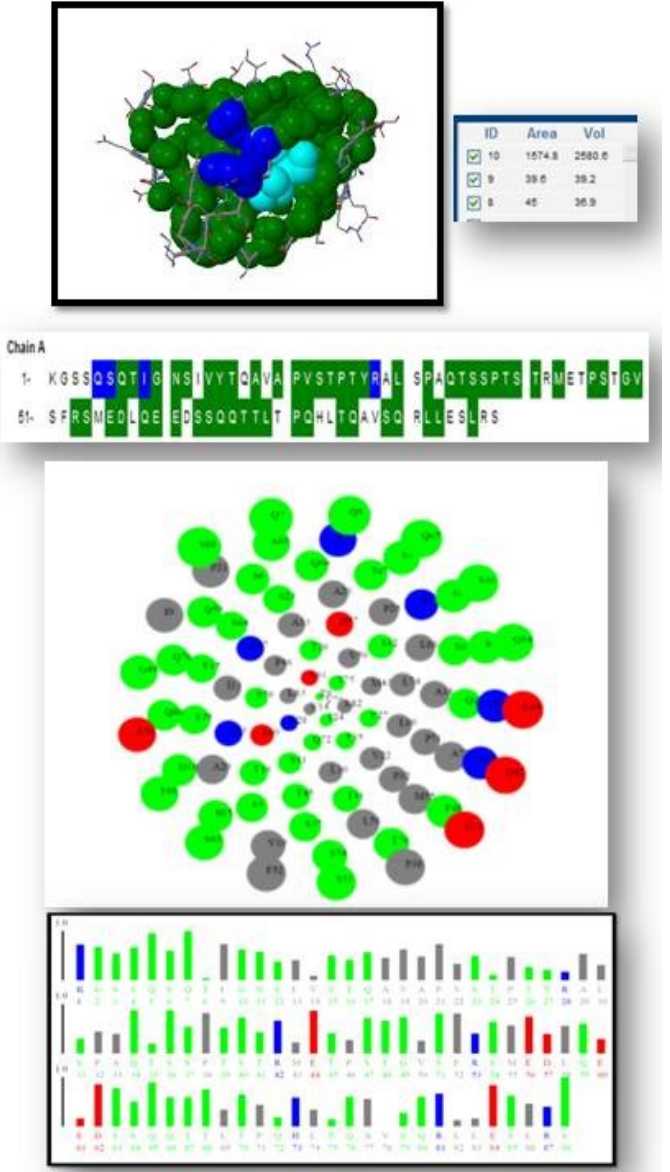

Fig. 2. Analyses of AC4 of SRILANKAN Cassava mosaic virus. 


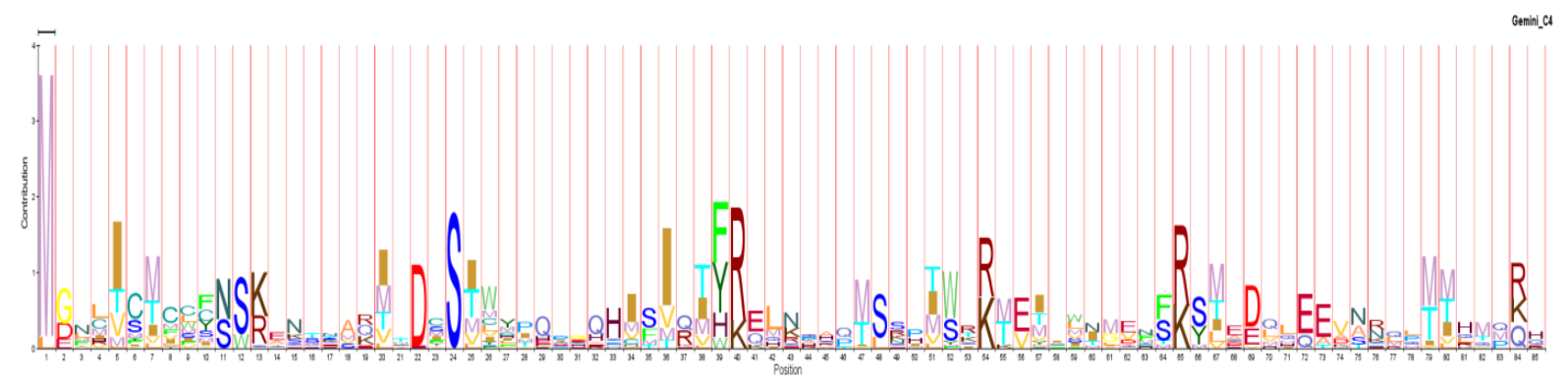

Fig. 3. Sequence logo for profile HMM for the multiple sequence alignment result (source Pfam).

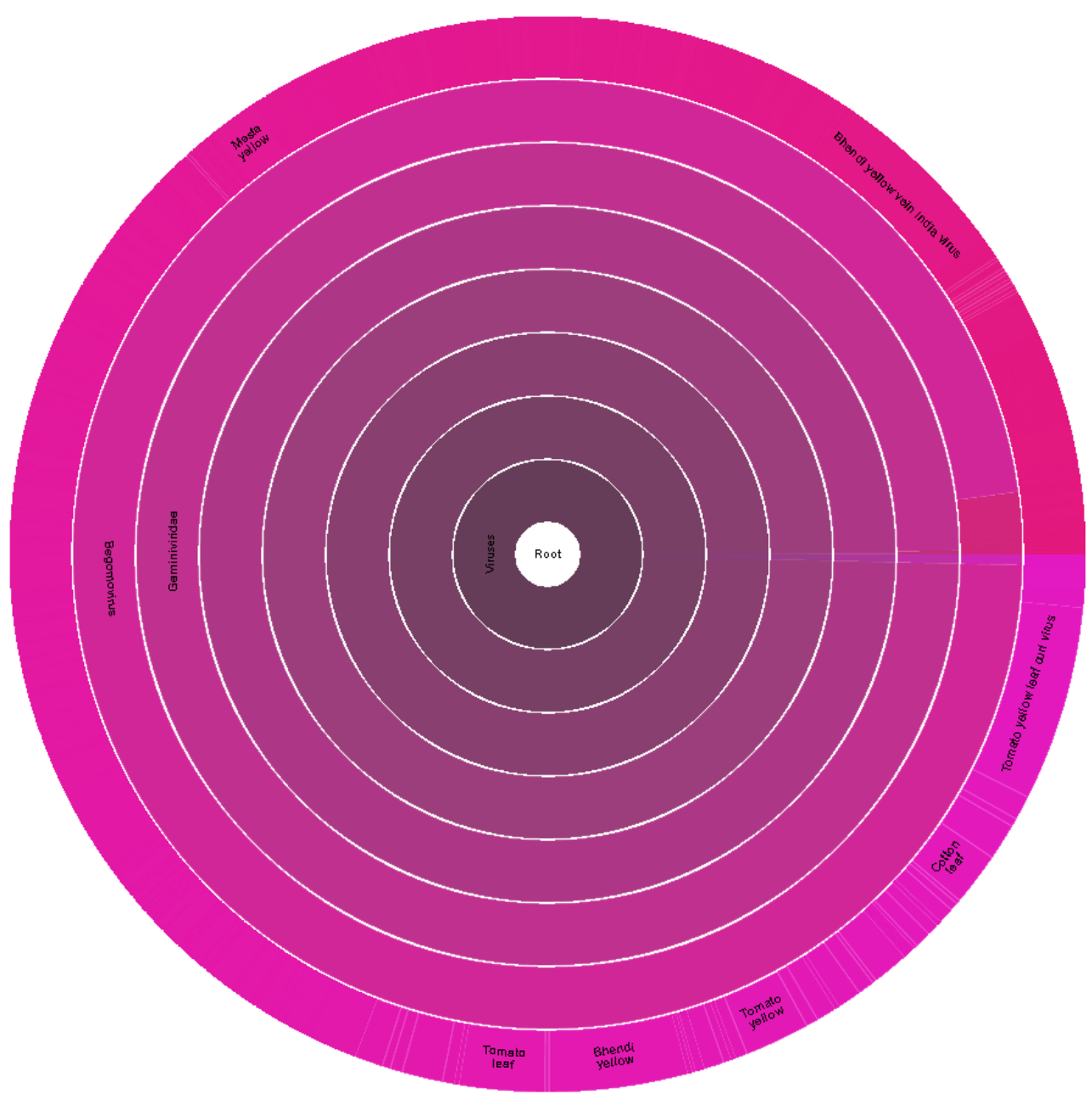

Fig. 4. Homologous species where AC4 protein is functional. This chart is a modified "sunburst" visualization of the species tree for this family. It shows each node in the tree as a separate arc, arranged radially with the superkingdoms at the centre and the species arrayed around the outermost ring. 
Till now more than 50 viral silencing suppressor proteins have been discovered. Experiments showed that the SCMV-encoded AC4, an RNA-silencing suppressor protein, binds to single-stranded miRNAs both in vitro and in vivo and inhibits miRNA-mediated negative regulation of gene expression in plants. Till date many RNA-silencing suppressors have been identified in plant viruses (Fig. 3) and these proteins are structurally and functionally diverse, and they likely target distinct steps of the RNA-silencing processes.

\section{CONCLUSION}

This analyses reveals that the structure of the AC4 viral silencing suppressor possess an ordered 3D structure with no specific disordered region. This 3D structure can be utilized for further studies on interactions with its cognate small RNA targets since this is the mode of action that has been assigned to AC4 proteins (Burgyán 2006).

\section{Acknowledgement}

The authors are indebted to the Department of Biotechnology, Government of India for the provision of funds for the maintenance of the DBT - Centre for Bioinformatics, Presidency University, Kolkata, under the BIF - BTBI scheme.

\section{References}

[1] Burgyán. J. Journal of Plant Pathology 88(3), (2006), 233-244.

[2] Chellappan, P. et al.. Journal of Virology. 78, (2004):7465-7477.

[3] Chellappan, P., Vanitharani, R. and Fauquet, C. M. Proc. Natl Acad. Sci. USA. 102 (2005):10381-10386.

[4] Elmer, J. S., Brand, L., Sunter, G., Gardiner, W. E., Bisaro, D.M., and Rogers, S. G. Nucleic Acids Res. 16 (1988): 7043-7060.

[5] Etessami, P., Saunders K., Watts, J., and Stanley, J. J Gen Virol. 72 (1991):1005-12.

[6] Fauquet, C. M., Bisaro, D. M., Briddon, R. W., Brown, J. K., Harrison, B.D., Rybicki, E. P. D., Stenger, C., and Stanley, J. Arch. Virol. 148 (2003): 405-421.

[7] Gutierrez C. Cell. Mol. Life Sci. 56 (1999): 313-329.

[8] Hoogstraten, R. A., Hanson, S. F., and Maxwell D. P. Mol. Plant-Microbe Interact. 9 (1996): 594-599.

[9] Pilartz, M., and Jeske, H. Virology. 189, (1992): 800-802.

[10]Pooma, W., and Petty, I. T. J. Gen. Virol. 77(1996): 1947-1951.

[11]Sung, Y. K., and Coutts, R. H. J. Gen. Virol. 76 (1995): 2809-15.

[12]Vanitharani, R., Chellappan, and Fauquet, C. M. Trends Plant Sci. Mar. 10(3) 2005: 14451. 
[13]Vanitharani, R., Chellappan, P., Pita, J. S., and Fauquet, C. M. J. Virol. 78 (2004): 94879498.

[14]Gupta. S, Ganguli. S and Datta. A, “Agricultural Bioinformatics” Eds: Kavi Kishore et.al, (2014) 21 - 32 (Chapter 4). 